\title{
Preliminary validation of the Portuguese Edinburgh Handedness Inventory in an adult sample
}

Helena Espírito-Santo, Catarina Freitas Pires, Inês Queiroz Garcia, Fernanda Daniel, Alexandre Gomes da Silva and Rachel L Fazio

\section{QUERY SHEET}

This page lists questions we have about your paper. The numbers displayed at left can be found in the text of the paper for reference. In addition, please review your paper as a whole for correctness.

Q1: Au: Please provide missing affiliation (Department).

Q2: Au: Please provide missing affiliation (Department).

Q3: Au: Please provide missing affiliation (Department).

Q4: Au: Please provide complete postal address.

Q5: Au: Please provide reference for citation [Crovitz \& Zener, 1962].

Q6: Au: Please provide reference for citation [Kline, 2005].

Q7: Au: Please provide reference for citation [Kline, 2005].

Q8: Au: Please confirm placement of the numbers presented in the horizontal column "Intellectual" in Table 1.

Q9: Au: Please provide reference for citation [Milenkovic, 2013].

Q10: Au: Citation [Dragovic, 2004] denotes [Dragovic, 2004a] or [Dragovic, 2004b]. Please check.

Q11: Au: Please provide reference for citation [Milenkovic, 2013].

Q12: Au: Please cite [Chang, 2014] in text or delete reference.

Q13: Au: Please cite [Cronbach, 1951] in text or delete reference.

Q14: Au: Please cite [Pallant, 2011] in text or delete reference.

Q15: Au: Please cite [Razali \& Wah, 2011] in text or delete reference.

Q16: Au: Please provide information for the sigificant 'a'. \& check the superscript variables are correctly set.

\section{TABLE OF CONTENTS LISTING}

The table of contents for the journal will list your paper exactly as it appears below:

Preliminary validation of the Portuguese Edinburgh Handedness Inventory in an adult sample Helena Espírito-Santo, Catarina Freitas Pires, Inês Queiroz Garcia, Fernanda Daniel,

Alexandre Gomes da Silva, and Rachel L Fazio 


\title{
Preliminary validation of the Portuguese Edinburgh Handedness Inventory in an adult sample
}

\author{
Helena Espírito-Santo ${ }^{a}$, Catarina Freitas Pires ${ }^{a}$, Inês Queiroz Garciaa ${ }^{a}$ Fernanda Daniela,b, \\ Alexandre Gomes da Silva ${ }^{c, b}$ and Rachel L Fazio ${ }^{d}$ \\ anstituto Superior Miguel Torga, Coimbra, Portugal; ${ }^{b}$ Centro de Estudos e Investigação em Saúde da Universidade de Coimbra, Coimbra, \\ Portugal; 'Instituto Politécnico de Coimbra., Coimbra, Portugal; ${ }^{\mathrm{C}} \mathrm{CAMH}$, Toronto, ON, Canada
}

\begin{abstract}
The Edinburgh Handedness Inventory (EHI) is persistently the most used inventory to evaluate handedness, being neuropsychological investigation and clinical practice. Despite this, there is no information on how this instrument functions in a Portuguese population. The objective of this study was therefore to examine the sociodemographic influences on handedness and establish psychometric properties of the $\mathrm{EHI}$ in a Portuguese sample. The sample consisted of 342 adults (157 men and 185 women), assessed with a battery of neuropsychological tests. The mean EHI Laterality Quotient was $63.52(S D=38.00)$. A much high percentage of ambiguous-handedness compared to left-handedness was detected. An inconsistency was found between the preference for formal education activities (writing-drawing-using scissors) and the remaining EHI activities. From sociodemographic variables, only age, area, and regions of residence showed significant influence on EHI scores. The reliability and temporal reliability of EHI were adequate. Confirmatory factor analysis indicated a one-factor model $\left(\chi^{2} / d f=2.141 ; T L I=0.972 ; C F I=0.979 ; R M S E A=\right.$ 0.058). The inconsistency between formal education and nonformal activities could be an indicator of social pressure. The present data give support for the notion that handedness measured by EHI is potentially sensitive to sociodemographic and cultural influences.
\end{abstract}

\section{KEYWORDS}

Edinburgh handedness; hand preference; handedness; inventory; psychometric properties

\section{Introduction}

Handedness is a broad concept involving a variety of neural and behavioral processes (Barbieri \& Gobbi, 2009; van Strien, 2002). Both genetic and epigenetic factors (i.e., environmental and cultural) influence handedness (Pogetti, De Souza, Tudella, \& Teixeira, 2013; Souza \& Teixeira, 2011). Specifically, by some estimates, genetic effects account for only about $24 \%$ of the variance in hand preference (Medland et al., 2009). Although it has not been determined exactly what percent is explained by the effects of culture, there are some indications it plays an important role. For example, van Strien (2002) found in his study that the choice of hand for writing is influenced by culture; the cultural differences demonstrated in the survey by Perelle and Ehrman (1994) are also quite striking. Considering that there is a social pressure for using the right hand, the environment likely plays a primary role in establishing laterality (Souza \& Teixeira, 2011).

Before delving further into these cultural phenomena, however, the methodological issue of the measurement of handedness and the effects of such must also be dealt with. It is well known that manual preference of humans is primarily classified into two distinct groups: having a dominant right hand (right-handed) or a dominant left hand (left-handed). However, in some cases, this dichotomy is accompanied by a third category: individuals who use both hands, whether this is indiscriminately or with set patterns of hand use for certain tasks (ambiguous-handedness and mixedhandedness) (Dragovic, Milenkovic, \& Hammond, 2008). To evaluate handedness, the two methods most commonly used include observation of the use of the dominant hand and the application of inventories answered by the individual (Barbieri \& Gobbi, 2009). The three most popular inventories (van Strien, 2002) are by Crovitz and Zener (1962), Annett (1970), and Oldfield (1971). The Edinburgh Handedness Inventory (EHI; Oldfield, 1971) is the most used from the previous three (Fazio \& Cantor, 2015; Veale, 2014). The EHI offers the advantage of being a simple and brief method of evaluating laterality using a quantitative scale (Oldfield, 1971).

The first version of the Oldfield Inventory was based on a modified version of the Humphrey inventory with 20 items (Büsch, Hagemann, \& Bender, 2010). There is 
evidence the original instructions and answer format were difficult to understand, and included problematic items (Fazio, Coenen, \& Denney, 2012; Oldfield, 1971; Veale, 2014). These issues have been improved over several versions (Veale, 2014). The most often used version of the Oldfield Inventory contains 10 items about handedness involving 10 motor tasks [writing, drawing, throwing, scissors, toothbrush, knife (without fork), spoon, broom (upper hand), striking match (match), opening box (lid)] (Büsch et al., 2010; Oldfield, 1971).

This study is dedicated to the EHI, given it is widely utilized in the determination of hand preference. As a small sample, the EHI has been studied in populations from England and Scotland (Oldfield, 1971), Canada (Bryden, 1977), Australia (Dragovic, 2004b), the United States (Messinger \& Messinger, 1995), and Serbia (Milenkovic \& Dragovic, 2013). The focus of these studies, however, has largely not included possible sociodemographic influences in handedness, potentially explaining a portion of the discrepancies in psychometric properties found amongst studies. There is a lack of information entirely regarding psychometric properties of the EHI for the Portuguese population; to fill this gap, this study is based on the Normative Studies of Neuropsychological Instruments project (ENIN, Estudos Normativos de Instrumentos Neuropsicológicos), developed at the Miguel Torga Institute.

The general goal is to study the psychometric properties of the EHI in an adult sample of the Portuguese population, in order to determine the rates of rightleft-, and other-handedness in this population. Other specific objectives included to: (a) Determine the descriptive statistics for the EHI; (b) Check the role of sociodemographic variables on EHI scores and handedness preference; (c) Analyze the reliability of the EHI (through Cronbach's alpha) and temporal stability (through testretest); and (d) Run a confirmatory factor analysis.

\section{Methods}

\section{Participants}

A total of 342 volunteers were included in the present analyses. The pre-study of power analysis through $\mathrm{G}^{\star}$ Power software (Buchner, Erdfelder, Faul, \& Lang, 2014; Faul, Erdfelder, Lang, \& Buchner, 2007a, 2007b) revealed this sample size was adequate to detect medium effects $(w=0,3 ; d=0,5 ; f=0,25 ; r=0,5)$ to get a power $>.95$, with alpha $=.05$ for the respective statistical tests (chi-square analysis, $t$-test, ANOVA, and correlation).

Participants were recruited among family, friends, and colleagues of students from neuropsychology classes of our Institute. Each student made a list of all potential participants, totaling 765 individuals. Afterwards prospective participants were randomly selected, with stratification for age, sex, and education in a total of 357 subjects. Of these, six people refused to participate $(1.7 \%)$. Participants did not receive any financial compensation for joining but were given the option to receive the results and their meaning if requested [64 participants asked for results (17.9\%)].

Present data were acquired as part of the ENIN. Because of that, the selection criteria included: (a) being able to read and write in Portuguese; (b) having Portuguese nationality or living in Portugal for more than 5 years; (c) having more than $50 \%$ of their education in Portugal; and (d) age between 18 and 65 . Based on these criteria, 15 volunteers were excluded because age was inferior to 18 years (4.2\%).

The participants in the sample were stratified according to their age, with stratification comprising six age groups: $18-19,20-29,30-39,40-49,50-59$, and 60-65 years. Participants were also stratified according to their educational level. Five educational groups were formed: the first cycle of basic education, the second cycle of basic education, the third cycle of basic education, secondary education, and higher education. ${ }^{1}$ The regions category was created according to Portuguese territorial units, where the South and the Autonomous Regions were merged for statistical analysis.

Finally, participants were classified into right-handed, ambiguous, and left-handed, in accordance with several authors who, in their studies about handedness, also opted for three categories (Fazio et al., 2012; Milenkovic \& Dragovic, 2013; Pogetti et al., 2013; van Strien, 2002; Veale, 2014).

\section{Procedures}

All participants filled out an informed consent form in accordance with the Helsinki Declaration. The EHI was administered as part of a neuropsychological battery. The tests were administered individually, in reserved areas without any distractive elements. The complete neuropsychological battery had a duration of 1 hour and took place between November 2014 and March 2015.

\section{Instruments}

The Edinburgh Handedness Inventory (Oldfield, 1971) determines hand preference (Büsch et al., 2010;

${ }^{1}$ The Portuguese school system comprises the first cycle of basic education which includes the 1st to 4th grade; the second cycle of basic education that includes the 5th to 6th grade; the third cycle of basic education with 7 th to 9th grades; secondary education with the 10th to 12th grade; and higher education that corresponds to university or college. 
Oldfield, 1971), and consists of ten items (writing, drawing, throwing, using scissors, a toothbrush, cutting with a knife, using spoon, the upper hand when using a broom, striking a match, and opening the lid of a box). On the response sheet, each item is followed by two columns labelled "left" and "right." The subject indicates " ++ " in the "left" or "right" column if they strongly prefer to use one hand for that task, " + " if the preference for using one hand is weak, and " + " in both columns if they are indifferent (Oldfield, 1971). Each " ++ " symbol is scored as 2 points and " + " as 1 point; therefore, the quotient of laterality may range between -100 (preference of "strong left") and +100 (preference of "strong right") and, finally, the formula is applied for the Laterality Quotient: $L Q=[(\mathrm{R}-\mathrm{L})$ / $(\mathrm{R}+\mathrm{L}) \times 100$ ] (Oldfield, 1971).

For the Portuguese EHI version, the guidelines proposed by Beaton, Bombardier, Guillemin, \& Ferraz (2000) were followed. A psychologist translated the EHI, and a person with no knowledge in the field of Psychology made a naïve translation. Both translations were compared, creating a consensual version with minor changes. Another psychologist back translated to English. Translation and back translation were compared, and the final version was created with no alterations.

\section{Statistical analysis}

For the analysis and processing of data, the statistical program Statistical Package for Social Sciences (SPSS Statistics, version 20.0 for Windows 10, SPSS, 2011) was used. For the preliminary analyses, descriptive statistics were computed for the EHI total scores, including means and standard deviations.

Using the cut-off of 60 for EHI LQ (Hardie \& Wright, 2014; Milenkovic \& Dragovic, 2013; Veale, 2014), handedness was classified as right-handed (100 to 61), ambiguous ( -60 to 60$)$, and left-handed ( -61 to -100 ). After this, frequencies and percentages were calculated and chi-square tests for goodness of fit were computed. To evaluate the association between each pair of handedness item, odds ratio (OR) were used (Agresti, 2010).

To explore the proportion of handedness cases that fall into each category of every sociodemographic variable, the chi-square test of independence (MantelHaenzsel linear-by-linear association/MH) or likelihood-ratio $\left(G^{2}\right)$ were computed as appropriate according to the variable's nature (Agresti, 2010). $T$-test/ANOVA was used to explore the effects of sociodemographic characteristics (age, sex, education, area of residence, region, and profession) on EHI scores. For the analysis of variance (ANOVA), the homogeneity of variances according to the Levene's test was determined. Whenever there was homogeneity $(p>.05)$, a Hochberg post hoc was used, otherwise a Games-Howell post hoc was used, both with the Bonferroni correction ( $p$ /number of pairwise comparisons) (Marôco, 2011).

For the analysis of the psychometric properties, internal consistency was determined by Cronbach's alpha. For test-retest analysis, Pearson's correlations $(r)$ and $t$-tests for paired samples $(2$-tailed; $p<.05)$ were computed. The $t$-test for paired samples was used to verify if the means between the two moments of evaluation were different or which one of them was higher.

The confirmatory factor analysis (CFA) with a maximum likelihood approach was performed using AMOS software version 23 (Arbuckle, 2014) taking in consideration the following aspects: (a) items were considered as categorical variables and analysed by polychoric correlations (Jöreskog, 1994); (b) as a parameter of fit estimation, the ratio of $\chi^{2}$ to the degrees of freedom $(d f)$ was computed, considering values lower than 3 as indicators of good fit of the model ( $\mathrm{Hu} \mathrm{\&}$ Bentler, 1999); (c) the Tucker-Lewis coefficient (TLI) was calculated as another measure of goodness of fit, with values over 0.90 indicating good fit ( $\mathrm{Hu} \&$ Bentler, 1999); (d) another parameter for fit estimation was the comparative fit index (CFI), with values larger than 0.90 indicating acceptable fit ( $\mathrm{Hu} \&$ Bentler, 1999; Kline, 2005); and (e) the root mean square error of approximation (RMSEA) was an additional index with values lower than 0.08 suggesting good fit $(\mathrm{Hu} \&$ Bentler, 1999; Kline, 2005).

Finally, the reliability of the construct was calculated using the composite reliability $(C R)$ estimate for the latent variable (Valentini \& Damásio, 2016), which should be greater than 0.7; Jöreskog, 1971b). The factorial validity was examined by analysing items' standardized weights, which, ideally, should exceed the recommended minimum of 0.60 (Bagozzi \& Yi, 1988); however, standardized weights between 0.5 and 0.6 are accepted when scales are applied in different contexts (Barclay, Higgins, \& Thompson, 1995). The mean extracted variance (MEV; Valentini \& Damásio, 2016) was used to assess how much variance in the measured items is captured by a latent construct (an acceptable level should be above 0.5; Fornell \& Larcker, 1981).

\section{Results}

\section{Sociodemographic}

Ages of participants ranged between 18 and 65 years old $(M=32.12, S D=13.43)$. Of the participants, $45.9 \%$ were men and $54.1 \%$ women. The time of their formal 
Table 1. Sociodemographic characterization $(N=342)$.

\begin{tabular}{|c|c|c|c|c|}
\hline & & $n$ & $\%$ & $x^{2, a} d f$ \\
\hline \multirow[t]{6}{*}{ Age $(M=32.12 ; S D=13.43)$} & $18-19$ & 19 & 5.6 & $389.09^{* * *} 5$ \\
\hline & $20-29$ & 191 & 55.8 & \\
\hline & $30-39$ & 35 & 10.2 & \\
\hline & $40-49$ & 38 & 11.1 & \\
\hline & $50-59$ & 44 & 12.9 & \\
\hline & $60-65$ & 15 & 4.4 & \\
\hline \multirow[t]{2}{*}{ Sex } & Men & 157 & 45.9 & $2.29^{\mathrm{NS}} 1$ \\
\hline & Women & 185 & 55.1 & \\
\hline \multirow[t]{5}{*}{ Education $(M=14.60 ; S D=3.96)$} & 1 st cycle of basic education & 10 & 2.9 & $336.45^{* * *} 4$ \\
\hline & 2nd cycle of basic education & 22 & 6.4 & \\
\hline & 3rd cycle of basic education & 19 & 5.6 & \\
\hline & Secondary education & 106 & 31.0 & \\
\hline & Higher education & 185 & 54.1 & \\
\hline \multirow[t]{3}{*}{ Area of residence } & Urban & 257 & 75.1 & $278.75^{* * *} 2$ \\
\hline & Transition Area & 19 & 5.6 & \\
\hline & Rural & 66 & 19.3 & \\
\hline \multirow[t]{4}{*}{ Regions } & North & 61 & 17.8 & $335.50^{* * *} 5$ \\
\hline & Center & 230 & 67.3 & \\
\hline & Lisbon & 24 & 7.0 & \\
\hline & South-Autonomous Regions & 27 & 7.9 & \\
\hline \multirow[t]{2}{*}{ Profession } & Manual & 37 & 12.1 & $175.90^{* * *} 1$ \\
\hline & Intellectual & 269 & 87.9 & \\
\hline
\end{tabular}

education ranged from 4 to 28 years of education ${ }^{2}$ $(M=14.49, S D=4.07)$.

Regarding area of residence, $75.1 \%$ of individuals

lived in urban areas, $5.6 \%$ in a transition area, and $19.3 \%$ in rural areas. As a result of recategorization, $17.8 \%$ lived in the North, $67.3 \%$ in the Centre, $7 \%$ lived in the Lisbon, and $7.9 \%$ in the South-Autonomous Regions. Concerning profession, $12.1 \%$ were in the manual category (i.e., workers) and $87.9 \%$ in the intellectual category (i.e., technical). Thirty-six individuals did not provide us any information regarding their profession. Full demographic information can be seen in Table 1.

\section{Preliminary analysis}

The mean EHI LQ was $63.52(S D=38.00)$. The distribution of EHI scores was negatively asymmetrical and leptokurtic. The Shapiro-Wilk test revealed a nonnormal distribution $(p<.001)$. Nevertheless, the skewness $(-1.44)$ and kurtosis (2.03) were within the Kim (2013) values; therefore, parametric statistical analyses were conducted. When using a cut-off of 60 for EHI LQ, of the participants, 59.1\% were righthanded (202 in number), 40.4\% were ambiguoushanded, and $0.6 \%$ reported being left-handed. Handedness was not equally distributed in the sample $\left[\chi^{2}(2, N=342)=183.02 ; p<.001\right]$.

\footnotetext{
${ }^{2}$ In the Portuguese educational system (pre-Bologna, 2005), high school took 12 years, a college degree 4 to 6 years (depending on the course), a master's degree 2 years, a doctoral degree 4 years, and a post-doctoral degree 3 years. Before 2005, it was not uncommon to take 3-4 years to get a master's degree and more than 4 years to a doctoral degree, and that is why two participants have 28 years of education. After 2006, a full education can still take 23 years without failing.
}

These results should be read in the light of what is true ambiguousness and mixed-handedness. True ambiguousness could be considered as having a high number of "either" responses or indifferent/inconsistent hand use within an item, and mixed-handed as having "left" and "right" responses or inconsistency across items (Fazio, Lykins, \& Cantor, 2014; Shaw, Claridge, \& Clark, 2001). Some have established a high number of "either" responses as two (Fazio et al., 2014), but others have referred to inconsistencies on three or more tasks (Satz, Nelson, \& Green, 1989). This calculation methodology is not well-established; therefore, some procedure variations were tested.

First, following Fazio et al.'s (2014) methodology, ambiguous-handedness was defined as having more or equal to two "either" responses. To start, the number of each type of response was calculated, resulting in five new continuous variables (designated strong right, weak right, strong left, weak left, and either). Because there were no weak responses, this kind of response was not considered. Then combinations of strong right, strong left, and either counts were considered resulting in new categories. As such, ambiguous-handedness corresponded to the following combinations: 2-10 "either" responses, $0-8$ "right" responses, and/or $0-8$ "left" responses; mixed-handed corresponded to combinations of 0-1 "either," 1-9 "right," and 1-9 "left responses (at least one "right" and one "left" must be present); right-handed to combinations 9-10 "right" and 0-1 "either"; and left-handed to combinations 9-10 "left" with 0-1 "either." As a consequence of this classification, $54.4 \%$ of the total sample was truly ambiguous, although $89.8 \%$ also had between 1 to 8 
"right" responses, and $14.0 \%$ had between 1 to 8 "left" responses. As mixed-handed, there were $11.4 \%$ participants, with $21.6 \%$ of them having 1 "either" response. Of those participants categorized as ambiguously handed (by means of cut-off of 60 ), 91.4\% were truly ambiguous and $8.6 \%$ mixed-handed. The two types of handedness categorization were significantly associated $(M H(1, N=342)=135.67 p<.001)$.

Second, using the number of items for which an "either" response was given as a continuous variable (Shaw et al. (2001) did the same for the Annett scale), a high number was having more or equal to five of the ten possible responses. In the resulting categories, true ambiguous-handed corresponded to 5-10 "either" responses, 0-5 "right" responses, and/or 0-5 "left" responses; mixed-handed to combinations of $0-4$ "either," 0-9 "right," and/or 0-9 "left responses (at least one "right" and one "left" must be present); right-handed to combinations 10-6 "right," 0-4 "either," or 0-1 "left"; and left-handed to combinations 6-10 "left" and 0-4 "either." In this classification, $27.5 \%$ of the total sample were truly ambiguous, among these $81.9 \%$ had between 1 and 5 "right" responses and $19.0 \%$ up to five "left" responses, and no one had 10 "either" responses. As mixed-handed there were $4.1 \%$ participants. Of participants categorized as ambiguously handed (cut-off of 60 ), $67.6 \%$ were truly ambiguous, $10.1 \%$ mixed-handed, $0 \%$ left-handed, and $22.3 \%$ right-handed. The two types of handedness categorization were significantly associated $(M H(1, N=342)=224.15, p<.001)$.

The third solution allowed "pure" categories to emerge, and true ambiguousness did not include any mixed handedness. As are result, for true ambiguousness the high number was considered whenever "either" counts surpassed the counts of the "right" or "left" responses (individually or added up), and for mixedhanded when the number of response type in two or three variables were equal, and if the same procedure is used, then new combinations of strong right, strong left, and either resulted in: ambiguous-handed corresponded to 6-10 "either" responses, 0-4 "right" responses, and/or 0-4 "left" responses; mixed-handed to combinations of $0-5$ "either", 0-9 "right," and/or 0-9 "left responses (at least one "right" and one "left" must be present). Then $19.3 \%$ were truly ambiguousness, with $81.8 \%$ also having between 1 and 4 "right" responses, and $18.2 \%$ between 2 to 4 "left" responses (there were no mixed answers). As mixed-handed there were $21.6 \%$ participants, with $58.1 \%$ of them having between 1 and 5 "either" response, 93.2\% had between 1 and 9 "right" responses, and 79.3\% had between 1 and 8 "left" responses. Ambiguously handed participants (EHI LQ cut-off of \pm 60 ) included $47.5 \%$ truly ambiguous, $33.8 \%$ mixed-handed, $0.7 \%$ left-handed, and $18.0 \%$ right-handed. Also, the two types of handedness categorization were significantly associated $(\mathrm{MH}$ $(1, N=342)=178.87, p<.001)$.

Given the results of the three solutions, for the purposes of the following analysis, "ambiguousness" will be used in sensu lato.

The item by item analysis (Table 2) shows that for the items broom and box, the percentages were slightly higher in the ambiguous category. Even on these two items, however, the observed proportion of participants who reported performing this activity with both the right hand and with the left hand (ambiguous) was very similar to the percentage of participants who reported performing this activity with only the right hand. For the items writing and drawing the lowest percentages of ambiguous were observed. These were items in which participants indicated more extreme responses, and it was where the highest percentages of right-handed and left-handed responses were found. Notably, the left-handed responses for these two items were the same people. The items broom and box were the other two items where the highest percentages of left-handers were found.

High positive associations were found for all the pairs of items $(p<.001)$. The highest associations occurred between the items writing and drawing $(O R=165.71)$, scissors and brush $(O R=129.94)$, scissors and spoon $(O R=110.77)$, scissors and striking match $(O R=123.48)$, brush and spoon $(O R=163.27)$,

Table 2. Frequency of types of handedness item by item of EHI $(N=342)$.

\begin{tabular}{|c|c|c|c|c|c|c|c|c|}
\hline \multirow[b]{2}{*}{ Items } & \multicolumn{2}{|c|}{ Right-handed } & \multicolumn{2}{|c|}{ Ambiguous } & \multicolumn{2}{|c|}{ Left-handed } & \multirow[b]{2}{*}{$\chi^{2, a}$} & \multirow[b]{2}{*}{$d f$} \\
\hline & $n$ & $\%$ & $n$ & $\%$ & $n$ & $\%$ & & \\
\hline Writing & 311 & 90.9 & 8 & 2.3 & 23 & 6.7 & $511.63^{* * *}$ & 2 \\
\hline Drawing & 314 & 91.8 & 4 & 1.2 & 24 & 7.0 & $528.07^{* * *}$ & 2 \\
\hline Throwing & 216 & 63.2 & 121 & 35.4 & 5 & 1.5 & $195.91^{* * *}$ & 2 \\
\hline Scissors & 276 & 80.7 & 57 & 16.7 & 9 & 2.6 & $355.42^{* * *}$ & 2 \\
\hline Tootbrush & 220 & 64.3 & 112 & 32.7 & 10 & 2.9 & $193.47^{* * *}$ & 2 \\
\hline Knife & 247 & 72.2 & 81 & 23.7 & 14 & 4.1 & $252.44^{* * *}$ & 2 \\
\hline Spoon & 222 & 64.9 & 110 & 32.2 & 10 & 2.9 & $197.33^{* * *}$ & 2 \\
\hline Broom & 150 & 43.9 & 162 & 47.4 & 30 & 8.8 & $93.47^{* * *}$ & 2 \\
\hline Striking Match & 223 & 65.2 & 105 & 30.7 & 14 & 4.1 & $192.65^{* * *}$ & 2 \\
\hline Box & 157 & 45.9 & 158 & 46.2 & 27 & 7.9 & $99.60^{* * *}$ & 2 \\
\hline
\end{tabular}


brush and knife $(O R=118.34)$, brush and striking match $(O R=133.94)$, spoon and knife $(O R=123.02)$, spoon and striking match $(O R=146.85)$, spoon and box $(O R=114.90)$, and knife and striking match $(O R=139.14)$.

\section{Role of sociodemographic variables}

Table 3 shows that regarding age, the highest percentages of right-handed individuals fall in the 18-19, 40-49, and 50-59 age categories; the frequencies showed minimal variation, but the linear-by-linear association was not statistical significant $(\mathrm{MH}$ $(1, N=342)=1.43 ; p=.232)$. Hand preference across ages varied in some activities: individuals ages 31-40 and 61-70 were equally likely to be classified as righthanded or ambiguous-handed in using a knife or a spoon ( $M H$ respectively $1.79, p<.05$; and $=12.63, p<.001$ ); $18-30,31-40$, and $61-70$ participants were more likely to be categorized as ambiguous-handed in opening a box $(M H(1, N=342)=17.10 ; p<.001)$.

Regarding sex, women were slightly more dexterous than men. However, the chi-square test for independence (linear-by-linear association) indicated no significant association between sex and handedness. The same was

likely to be classified as right-handed or ambiguoushanded, and males more likely to be classified as ambigu-
For education, the proportion of right-handers was larger in the first, second, and third cycle of basic education than secondary education and higher education. In secondary education and higher education, the percentages of right-handed and ambiguous were similar. There was no association between education and hand preference across the ten items $(p>.05)$. Regarding profession, right-handedness prevailed in the manual category, but there was no association with each EHI item ( $G^{2}$ between .20 and 3.65, $\left.p>.05\right)$.

Concerning residence area, there was a greater number of right-handers in the transition area; in rural areas there were more ambiguous individuals than right-handed. Regarding the regions, a greater proportion of ambiguousness was observed both in Lisbon and in the South-Autonomous Regions compared to the North and the Center where the population appears more right-handed. The same happened for all activities $\left(G^{2}\right.$ between 18.20, $p<.01$ and 74.04, $p<.001)$.

These observations fell mostly in the right-handed and ambiguous categories, as there were only two subjects in the left-handed category; it was therefore not feasible to perform comparison to other categories. As is apparent from Table 3, these individuals were in the age groups of 20-29 and 40-49 years old, a woman and a man who completed the second cycle of basic education and higher education, resided in the urban areas of the North regions, and practiced an intellectual profession.

Table 4 illustrates the differences in EHI LQ mean scores between groups defined by sociodemographic ous-handed $\left(G^{2}(2, N=342)=6.42, p<.05\right)$.

Table 3. Frequency of types of handedness by sociodemographic variables $(N=342)$.

\begin{tabular}{|c|c|c|c|c|c|c|c|c|}
\hline & & \multicolumn{2}{|c|}{ Right-handed } & \multicolumn{2}{|c|}{ Ambiguous } & \multicolumn{2}{|c|}{ Left-handed } & \multirow[b]{2}{*}{$\chi^{2 a}$} \\
\hline & & $n$ & $\%$ & $n$ & $\%$ & $N$ & $\%$ & \\
\hline \multirow[t]{6}{*}{ Age } & $18-19$ & 14 & 73.7 & 5 & 26.3 & 0 & 0 & $6.58^{*}$ \\
\hline & $20-29$ & 97 & 50.8 & 93 & 48.7 & 1 & 0.5 & \\
\hline & $30-39$ & 20 & 57.1 & 15 & 42.9 & 0 & 0 & \\
\hline & $40-49$ & 27 & 71.1 & 10 & 26.3 & 1 & 2.6 & \\
\hline & $50-59$ & 36 & 81.8 & 8 & 18.2 & 0 & 0 & \\
\hline & $60-65$ & 8 & 53.3 & 7 & 46.7 & 0 & 0 & \\
\hline \multirow[t]{2}{*}{ Sex } & Men & 88 & 56.1 & 68 & 43.3 & 1 & 0.6 & $1.07^{\mathrm{NS}}$ \\
\hline & Women & 114 & 61.6 & 70 & 37.8 & 1 & 0.5 & \\
\hline \multirow[t]{5}{*}{ Education } & 1st CBE & 7 & 70.0 & 3 & 30.0 & 0 & 0 & $1.53^{\mathrm{NS}}$ \\
\hline & 2nd CBE & 15 & 68.2 & 6 & 27.3 & 1 & 4.5 & \\
\hline & 3rd CBE & 14 & 73.7 & 5 & 26.3 & 0 & 0 & \\
\hline & Secondary education & 60 & 56.6 & 46 & 43.4 & 0 & 0 & \\
\hline & Higher education & 106 & 57.3 & 78 & 42.2 & 1 & 0.5 & \\
\hline \multirow[t]{3}{*}{ Area of Residence } & Urban & 156 & 60.7 & 99 & 38.5 & 2 & 0.8 & $2.74^{\mathrm{NS}}$ \\
\hline & Transition Area & 16 & 84.2 & 3 & 15.8 & 0 & 0 & \\
\hline & Rural & 30 & 45.5 & 36 & 54.5 & 0 & 0 & \\
\hline \multirow[t]{4}{*}{ Regions } & North & 46 & 75.4 & 14 & 23.0 & 1 & 1.6 & $46.86^{* * *}$ \\
\hline & Center & 151 & 65.7 & 78 & 33.9 & 1 & 0.4 & \\
\hline & Lisbon & 3 & 12.5 & 21 & 87.5 & 0 & 0 & \\
\hline & SAR & 2 & 7.4 & 25 & 92.6 & 0 & 0 & \\
\hline \multirow[t]{2}{*}{ Profession } & Manual & 26 & 70.3 & 10 & 27.0 & 1 & 2.7 & $1.00^{\mathrm{NS}}$ \\
\hline & Intellectual & 159 & 59.1 & 109 & 40.5 & 1 & 0.4 & \\
\hline
\end{tabular}

Notes. $\mathrm{CBE}=$ Cycle of Basic Education; SAR $=$ South and Autonomous Regions. ${ }^{a}$ Linear-by-linear association ${ }^{*} p<.05$. ${ }^{* * *} p<.001 ;{ }^{\text {NS }}$ Statistically nonsignificant. 
Table 4. Differences in scores of EHI between categories of the sociodemographic variables $(N=342)$.

\begin{tabular}{|c|c|c|c|c|c|}
\hline & & $n$ & $M \pm S D$ & Cl 95\% IL - UL & Range Min-Max \\
\hline \multirow[t]{6}{*}{ Age $F(5,336)=3.64 p<.01 \eta^{2}=0.05$} & $18-19$ & 19 & $73.68 \pm 37.74$ & $55.49-91.88$ & $-50-100$ \\
\hline & $20-29$ & 191 & $57.54 \pm 38.71$ & $52.01-63.06$ & $-80-100$ \\
\hline & $30-39$ & 35 & $60.00 \pm 41.16$ & $45.86-74.14$ & $-50-100$ \\
\hline & $40-49$ & 38 & $73.16 \pm 38.70$ & $60.44-85.88$ & $-80-100$ \\
\hline & $50-59$ & 44 & $80.23 \pm 22.15$ & $73.49-86.96$ & $20-100$ \\
\hline & $60-65$ & 15 & $60.00 \pm 39.10$ & $38.35-81.65$ & $-20-100$ \\
\hline \multirow[t]{2}{*}{$\operatorname{Sex} t(340)=1.49 p>.05$} & Men & 157 & $60.13 \pm 38.21$ & $54.26-66.31$ & $-60-100$ \\
\hline & Women & 185 & $66.27 \pm 37.60$ & $60.82-71.72$ & $-80-100$ \\
\hline \multirow[t]{5}{*}{ Education $F(4,337)=0.68 p>.05 \eta^{2}=0.008$} & 1st CBE & 10 & $81.00 \pm 26.85$ & $61.79-100.21$ & $40-100$ \\
\hline & 2nd CBE & 22 & $64.55 \pm 42.40$ & $45.75-83.34$ & $-80-100$ \\
\hline & 3rd CBE & 19 & $68.42 \pm 41.67$ & $48.34-88.50$ & $-50-100$ \\
\hline & Secondary Education & 106 & $63.30 \pm 39.44$ & $55.71-70.90$ & $-60-100$ \\
\hline & Higher Education & 185 & $61.95 \pm 37.95$ & $56.62-67.49$ & $-80-100$ \\
\hline \multirow[t]{3}{*}{ Area of Residence $F(2,339)=3.30 p<.05 \eta^{2}=0.02$} & Urban & 257 & $64.63 \pm 37.54$ & $60.02-69.24$ & $-80-100$ \\
\hline & Transition Area & 19 & $77.89 \pm 24.40$ & $66.13-89.65$ & $0-100$ \\
\hline & Rural & 66 & $54.70 \pm 41.18$ & $44.57-64.82$ & $-60-100$ \\
\hline \multirow{4}{*}{ Regions $F(3,338)=19.71 p<.001 \eta^{2}=0.15$} & North & 61 & $71.48 \pm 36.46$ & $62.14-80.81$ & $-80-100$ \\
\hline & Center & 230 & $69.04 \pm 35.24$ & $64.46-73.62$ & $-80-100$ \\
\hline & Lisbon & 24 & $30.42 \pm 30.57$ & $17.51-43.33$ & $-30-80$ \\
\hline & SAR & 27 & $27.04 \pm 35.28$ & $13.08-40.99$ & $-50-100$ \\
\hline \multirow[t]{2}{*}{ Profession $t(304)=0.63 p>.05$} & Manual & 37 & $59.73 \pm 49.24$ & $43.31-76.15$ & $-80-100$ \\
\hline & Intellectual & 269 & $64.07 \pm 37.61$ & $59.56-68.59$ & $-80-100$ \\
\hline
\end{tabular}

Notes. $M=$ Mean; $S D=$ Standard Deviation; $C I$ 95\% = Confidence Interval 95\%; $I L=$ Inferior Limit; UL = Upper Limit; Min = Minimum; Max = Maximum; $F=$ ANOVA; $t=$ Student $t$ test; $p=$ level of statistical significance; $\eta^{2}=$ eta-squared; $C B E=$ Cycle of Basic Education; SAR $=$ South and Autonomous Regions.

variables with $t$-test/ANOVA values as appropriate. The scores on the EHI LQ differed significantly among the six age groups $\left[F(5,336)=3.64 ; p<.01 ; \eta^{2}=0.05\right]$. Regarding sex, there were no statistically significant differences $[t(340)=1.49 ; p=.136$; Cohen's $d=0.16]$. Level of education had no influence on EHI LQ $\left[F(4,337)=0.69 ; p=.598 ; \eta^{2}=0.008\right]$. With regard to the three residential areas, the scores on the EHI were statistically different $[F(2,339)=3.30 ; \quad p<.05$; $\left.\eta^{2}=0.02\right]$. The scores on the EHI LQ differed significantly among the four regions as well $[F(3,338)=$ $\left.19.71 ; p<.001 ; \eta^{2}=0.15\right]$. Profession did not influence EHI LQ $[t(304)=0.51 ; p=.62$; Cohen's $d=0.10]$.

The Hochberg post hoc test (Table 5) with Bonferroni correction indicated differences between age category 20-29 and 50-59 with EHI LQ, with there being more ambiguous in the first and more right-handed in the 50-59 category. About the area of residence, the Games-Howell post hoc test showed differences between the rural and transition area for EHI LQ. As for the regions, the Hochberg post hoc test was used with the Bonferroni correction, where differences were found between the North and Lisbon; the North and SouthAutonomous Regions; the Centre and Lisbon; and the Center and the South-Autonomous Regions for EHI LQ. There were more right-handed people in the North than Lisbon and South-Autonomous Regions; in Lisbon and South-Autonomous Regions, ambiguousness prevailed.

To check if these results could be explained by potential differences in education or profession among area of residence and regions, correlations were computed between EHI LQ scores and those variables in the subgroups defined by area/regions. For education, the only significant correlation was found with EHI LQ in the North region $(r=-0.28 ; p<.05)$, where the level of education was significantly lower $(M=12.67$; $S D=4.28$ ). For profession, participants with manual occupations had higher EHI LQ scores in the transition areas $(r=-0.51 ; p<.05)$.

Table 5. Post-hoc comparisons of the scores of EHI in which there were differences by sociodemographic variables.

\begin{tabular}{|c|c|c|c|c|c|c|}
\hline Variables & & & $M$ Difference & $p$ & $d$ & $d$ Interpretation \\
\hline $\begin{array}{l}\text { Age } \\
F(5,336)=3.64 \\
p<.01\end{array}$ & $\begin{array}{l}20-29 \\
M=57.54 \\
S D=37.74\end{array}$ & $50-59$ & 22.69 & .005 & 0.73 & Medium Effect \\
\hline $\begin{array}{l}\text { Area of Residence } \\
F(2,339)=3.30 \\
p<.05\end{array}$ & $\begin{array}{l}\text { Rural } \\
M=55.70 \\
S D=41.18\end{array}$ & Transition Area & 23.20 & .011 & 0.66 & Medium Effect \\
\hline Regions & North & Lisbon & 41.06 & $<.001$ & 1.22 & Large Effect \\
\hline $\begin{array}{l}F(3,338)=19.80 \\
p<.001\end{array}$ & $\begin{array}{l}M=71.48 \\
S D=36.46\end{array}$ & SAR & 44.44 & $<.001$ & 1.24 & Large Effect \\
\hline & $\begin{array}{l}\text { Center } \\
M=69.04 \\
S D=35.24\end{array}$ & $\begin{array}{l}\text { Lisbon } \\
\text { SAR }\end{array}$ & $\begin{array}{l}38.63 \\
42.01\end{array}$ & $\begin{array}{l}<.001 \\
<.001\end{array}$ & $\begin{array}{l}1.17 \\
1.19\end{array}$ & $\begin{array}{l}\text { Large Effect } \\
\text { Large Effect }\end{array}$ \\
\hline
\end{tabular}

Notes. $\mathrm{M}=$ Mean; $S D=$ Standard Deviation; $p=$ level of statistical significance; $d=$ Cohen's $d ; S A R=$ South and Autonomous Regions. 


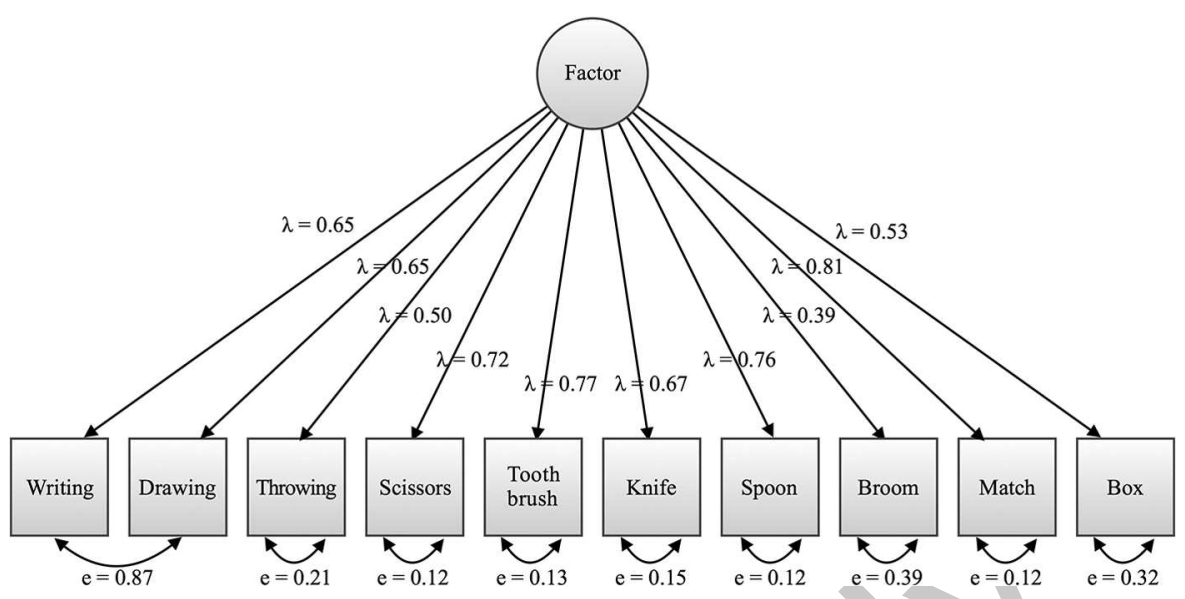

Figure 1. Confirmatory factor analysis with a maximum likelihood approach of the Edinburgh Handedness Inventory ( $\lambda=$ standardized factor loadings; e = error variances), Model fit: Ratio of chi-square to degrees of freedom $=2.141$; Tucker-Lewis coefficient $=0.972$; Comparative fit index $=0.979$; Root mean square error of approximation $=0.058$.

\section{Psychometric properties}

Reliability. Concerning the internal consistency of the EHI, Cronbach's alpha was 0.877 . This value is considered good for research purposes (Pestana \& Gageiro, 2008), meaning that the scale has good internal consistency.

Test-retest reliability. To determine the temporal stability of EHI, the test was re-administered to a group of 25 individuals after a period of 5 months $(M=4.96$; $S D=1.34)$. Through $t$-test it was found that there were no statistically significant differences for the EHI LQ $[t(24)=0.37 ; p>.05]$ between the first and the second moment, and the effect size was insignificant (Cohen's $d=0.02$ ). Pearson correlations confirmed a high positive correlation $(r=0.97 ; p<.001)$ (Pestana \& Gageiro, 2008). The same happened for the individual items, with correlations varying between 0.59 ( $p<.01$; opening box) and 1.00 (writing, drawing, using scissors) $(p<.001)$.

Factor analysis. Since there was collinearity between the writing and drawing items $(r=0.94)$, an adjustment was made through the establishment of a correlation between the error variance of those items, and the resulting model (Figure 1) was adequate $\left(\chi^{2} / d f=2.141 ; T L I=0.972\right.$; $C F I=0.979 ;$ RMSEA $=0.058 ; p[R M S E A<.05]<.001)$.

Analyzing the reliability of the model through the composite reliability, we verified that $C R$ was 0.91 . The factorial validity was also appropriate since almost all items had standardized factor loadings $>0.5$ and square factor loadings $>0.25$ (the broom item had a factor loading close to adequate). The mean extracted variance was good $(M E V=0.61)$.

\section{Discussion and conclusion}

The purpose of the present study was to investigate the psychometric properties of the Edinburgh Handedness
Inventory (EHI). From the descriptive statistics, we observed only two left-handed participants in our sample. Interestingly, according to the item-by-item analysis, we found there were 23 participants that reported performing the activity writing only with the left hand; the same participants (except for one) also indicated drawing only with the left hand. Our data suggests that there is a possibility of social pressure to be right-handed, in line with several studies (e.g., Christman, Prichard, \& Corser, 2015; van Strien, 2002), which state that hand preference may be related to culture/environment, and that low numbers of lefthanders could be explicable by cultural and biological differences among samples (Salmaso \& Longoni, 1985). As a result, the present data indicates the conformity pressures, brain, and behavioral lateralization may have grown under social selection pressures (Ghirlanda \& Vallortigara, 2004; Payne, 1987). Giving strength to this hypothesis is the inconsistency found in the preference for writing-drawing-using scissors (activities more related to formal education) and other activities (using knife, match, spoon, toothbrush, broom, throwing, and opening box). Formal education activities are more prone to forced correction than the other activities, and congruency between these two types of activities might be an indicator of forced correction decreasing (Lai, Serra, Petretto, Masala, \& Preti, 2014; Merni, Di Michele, \& Soffritti, 2014). Another finding reinforcing this hypothesis is the unusually high percentage of ambiguous-handedness (which was much higher than left-handedness in this sample).

The prevalence of ambiguous-handedness raises other reflections. No matter the calculation procedure used in the present study, the rates are relatively high (between 19.3\% and 54.4\%), not substantiating early 
hypotheses that ambiguous-handedness is rare among normal populations (Satz et al., 1989: 3-4\%), and presumed to be pathological (e.g., Bolinskey, Iati, Hunter, \& Novi, 2013; Fazio et al., 2014; Lange et al., 2010; Shaw et al., 2001; Soper \& Satz, 1984; Tsuang, Chen, \& Kuo, 2013). Nonetheless, a note of caution should be made: the left/right/ambiguous classification schemes vary largely due to different cutoff scores, as a review of 899 papers published from 1998 to 2012 has shown, making difficult the basic left-right differentiation and hampering rate comparisons between studies (Edlin, Leppanen, Fain, \& Hackländer, 2015).

Regarding the effect of sociodemographic variables on the performance of EHI, the results showed that concerning the six sociodemographic variables (age, sex, education, area of residence, region, and profession) three influenced EHI scores: age, area of residence, and region. Regarding age, we found significant differences, which is also consistent with Milenkovic and Dragovic (2013). There were differences between the category 20-29 and 50-59. The fact that there are more ambiguous in the 20-29 age group and more righthanded in the 50-59 category allows one to conclude that social pressure to be right-handed it is not as strong as it was in the past, but it still does not follow the increasing trend of left-hand preference in the younger generations reported by others (McManus, Moore, Freegard, \& Rawles, 2010; Preti et al., 2011). This is somewhat different from Medland, Perelle, Monte De, \& Ehrman (2004), where older participants were more likely to be classified as left-handed. This disparate evidence reinforces the idea of cultural pressure as a shifting influence upon handedness.

In contrast to studies by Bryden (1977), Oldfield (1971), Martin, Papadatou-Pastou, Jones, and Munafò (2010), Medland et al. (2004), and Papadatou-Pastou, Martin, Munafò, and Jones (2008) which showed a greater propensity for men to be more left-handed than women, and distinguished from the meta-analytic study of Papadatou-Pastou et al. that reported a greater tendency of mixed-handedness among males, we found no differences in scores on the EHI between men and women. This absence of differences may also reflect the influence of cultural factors given the overall very low prevalence of left-handedness in this sample. Although the level of left-handedness was much higher in the Preti et al. (2011) study (c. 10\%), they also found we found some differences, which may reflect, again, a cultural influence.

We also did not find differences between education and scores on the EHI which was in contrast to Fazio et al. (2012), where years of school was a significant predictor, since participants with a higher education level tend to read and follow instructions better. It should be noted though that a correlation between education and EHI scores in the Northern region was found. According to Portuguese census (PORDATA, 2015) this region is similar to the other main regions (Center and Lisbon) insofar as education is concerned, which was not the case in our study. Giving the potential for some selection bias, this finding should be confirmed with more representative samples.

Despite the absence of studies that address the variable of area of residence, we decided to include it in our study. We found differences between rural and transition areas, which is also consistent with Fagard and Dahmen (2010), Leask and Beaton (2007), Medland et al. (2004), and van Strien (2002) on the binding of hand preference with culture. As with the aforementioned variable, the same happens with the regions. By observing the Northern average falls into the righthanded category compared with the other groups that fall into the ambiguous category, it may reinforce the suggestion that there is actually an interference of culture and pressure, in this case by the inhabitants of the North, to be right-handed. The same explanation was drawn by Greenwood, Greenwood, McCullagh, Beggs, and Murphy (2006), Leask and Beaton (2007), and Viggiano, Borelli, Vannucci, and Rocchetti (2001) that there are potential regional differences in tolerance for left-handedness. Geographical variations in disease (e.g., psychosis) or developmental conditions (e.g., developmental coordination disorder) can also be invoked as possible explanations (Dragovic \& Hammond, 2005; Goez \& Zelnik, 2007; Leask \& Beaton, 2007). Nevertheless, we should not forget that there was a significant correlation between handedness and education only in the North region.

Regarding profession, no differences in scores were found on the EHI by the categories of this variable; there appear to be no published studies that support or contrast with these results.

Milenkovic (2013) previously used confirmatory factor analysis to examine the psychometric properties of the EHI, and the results showed the EHI has poor psychometric properties. In contrast in this study, we obtained good results both on internal consistency and on temporal stability. Studies of Büsch et al. (2010) and Veale (2014) rejected the one-dimensionality of the construct. However, from our confirmatory factor analysis, the 1-factor model showed adequacy, as in the van Strien (2002) study, which by principal component analysis (albeit with a version of EHI which contained
645 
16 items), revealed a single dimension of laterality. The studies of Williams (1986), McFarland \& Anderson (1980), Fazio \& Cantor (2015), and Richardson (1978) also obtained one factor. Dragovic (2004) obtained a one-factor solution at expenses of removing two of the items, and we obtained a one-factor solution accounting for the inter-item correlation by adjusting the model through the establishment of a correlation between the error variance of writing and drawing items. Again, this suggests a cultural component in this way of evaluating the handedness, and as Medland et al. (2004) indicate, there are some measures of handedness more sensitive to cultural influences. Additionally, the different factorial solutions could be a consequence of different interpretations of the items which also point to a cultural influence (Jöreskog, 1971a; Millsap, 2012), or to the difficulty following the instructions (Fazio et al., 2012).

Since level of education predicts ability to follow the EHI instructions (Fazio et al., 2012), in future studies measurement invariance by education level should be tested. It is also worth noting that the samples' nature and statistical procedures varied across studies (Dragovic, 2004b: mean age 44.8 to 47.4 , SEM with LISREL; McFarland \& Anderson, 1980: students, principal component analysis; Milenkovic, 2013: students, confirmatory factor analysis; Büsch et al., 2010: ages between 17-37 years, mixed-Rasch analyses; Williams, 1986: students, principal component analysis). Moreover, the item-oriented approach inherent to the CFA might have contributed to the failure of the model in some studies (Büsch et al., 2010).

There were some minor methodological limitations to this study. The first relates to education, which was operationalized as the number of years of regular formal education successfully completed; this approach is vulnerable to the numerous changes in the Portuguese education system. This limitation has been minimized, however, by the large size of the sample and the absence of significant differences between the various educational groups. During the administration of the EHI, some participants showed difficulties in understanding the test instructions, similar to the experience of other authors (Fazio et al., 2012; Oldfield, 1971; Veale, 2014). We suggest that, in future, the answers of EHI should be collected orally or replaced with a five-checkbox system (e.g., with strong left on the left, neutral in the middle, and strong right on the far right). Another limitation concerns the handedness classification established with cut-off of an LQ of 60 which is not entirely consistent with classifications based on number of "either", "left" or "right" answers. Others also have reported misclassification of cases (Dragovic, 2004a; Fazio et al., 2014). In future studies, it is proposed, in accordance to Dragovic (2004a), to classify handedness based on statistical criteria and use a model based approach (latent class analysis).

Finally, the main limitation of this study was the fact that the sample was not representative of the Portuguese population. The distribution of percentages in most of the sociodemographic variables was not according to the last census conducted in Portugal (Instituto Nacional de Estatística, 2014). A large percentage of participants were between the ages of 20 to 29 years; there were also a large percentage of participants with higher education. The same applies to the area of residence and regions: the urban area and the central region were over-represented in comparison to other categories. This limitation may be the result of the sampling type that was used (non-probability sampling). In future studies, we suggest using a random sampling method, to better generalize the results (Hill \& Hill, 2000).

In conclusion, although analyzing sociodemographic influence is not a means to directly evaluate cultural influence on EHI, results suggest that handedness measured by EHI is potentially sensitive to cultural influences, even though it is a measure with preliminary evidences of adequate internal consistency, temporal stability, and construct validity. A warning for studies of handedness in Portugal and perhaps other countries should be added: incidence of hand preference from one region/area of the country should not be compared with data from another region/area without a consideration of the potential cultural or biological factors. More generally, for the practicing neuropsychologist, this study serves two main purposes. The first is to establish the EHI as a valid measure for evaluating handedness with individuals from Portugal. The second is to provide information regarding the difference in lateralization amongst individuals from these areas. In the setting of pre-surgical epilepsy evaluations or other important neurological evaluations of language lateralization, the EHI scores obtained may not be adequate for this purpose in non-North American individuals. Future research correlating EHI scores to fMRI results would be illustrative in this scenario.

\section{Acknowledgments}

The authors thank Alexandra Mós, Débora Domingues, and Inês Pessoa for their help with recruitment and coding the data. The authors would also like to thank Rita Carvalho, Diogo Oliveira and Bruno Carvalho for their help in the translation and translation review of the article. 


\section{References}

Agresti, A. (2010). Analysis of ordinal categorical data. Hoboken, NJ: Wiley.

Annett, M. (1970). A classification of hand preference by association analysis. British Journal of Psychology, 61, 303-321. doi:10.1111/j.2044-8295.1970.tb01248.x

Arbuckle, J. L. (2014). IBM SPSS ${ }^{\circledR}$ Amos $^{T M} 23$ user's guide. Amos Development Corporation. Retrieved from ftp:// public.dhe.ibm.com/software/analytics/spss/documentation/ amos/23.0/en/Manuals/IBM_SPSS_Amos_User_Guide.pdf.

Bagozzi, R. P., \& Yi, Y. (1988). On the evaluation of structural equation models. Journal of the Academy of Marketing Science, 16(1), 74-94. doi:10.1007/BF02723327

Barbieri, F. A., \& Gobbi, L. T. B. (2009). Assimetrias laterais no movimento de chute e rendimento no futebol e no futsal [Lateral asymmetry in the kicking motion and performance in football and futsal]. Motricidade, 5(2), 33-47. doi:10.6063/motricidade.5(2).180

Barclay, D., Higgins, C., \& Thompson, R. (1995). The partial least squares (PLS) approach to causal modelling: personal computer adoption and use as an illustration. Technology Studies, 2(2), 285-309.

Beaton, D. E., Bombardier, C., Guillemin, F., \& Ferraz, M. B. (2000). Guidelines for the process of cross-cultural adaptation of self-report measures. Spine, 25(24), 3186-3191. doi:10.1097/00007632-200012150-00014

Bolinskey, P. K., Iati, C. A., Hunter, H. K., \& Novi, J. H. (2013). Season of birth, mixed-handedness, and psychometric schizotypy: Preliminary results from a prospective study. Psychiatry Research, 208(3), 210-214. doi:10.1016/ j.psychres.2012.12.023

Bryden, M. P. (1977). Measuring handedness with questionnaires. Neuropsychologia, 15(4-5), 617-624. doi:10.1016/ 0028-3932(77)90067-7

Buchner, A., Erdfelder, E., Faul, F., \& Lang, A.-G. (2014). $G^{*}$ Power 3 (Version 3.1.9.2 for Macintosh) [Informatic software]. Düsseldorf, Germany: Heinrich-Heine-Universität Dusseldorf. Retrieved from http://www.gpower.hhu.de/en. html.

Büsch, D., Hagemann, N., \& Bender, N. (2010). The dimensionality of the Edinburgh Handedness Inventory: An analysis with models of the item response theory. Laterality, 15(6), 610-628. doi:10.1080/13576500903081806

Chang, A. (2014). StatsToDo [Online calculator]. Brisbane, Queensland, Australia. Retrieved from http://www. statstodo.com/SSiz1Alpha_Pgm.php\#

Christman, S. D., Prichard, E. C., \& Corser, R. (2015). Factor analysis of the Edinburgh Handedness Inventory: Inconsistent handedness yields a two-factor solution. Brain and Cognition, 98, 82-86. doi:10.1016/j.bandc.2015.06.005

Cronbach, L. J. (1951). Coefficient alpha and the internal structure of testes. Psychometrika, 16, 297-334. doi:10.1007/bf02310555

Dragovic, M. (2004a). Categorization and validation of handedness using latent class analysis. Acta Neuropsychiatrica, 16(4), 212-218. doi:10.1111/j.0924-2708.2004. 00087.x

Dragovic, M. (2004b). Towards an improved measure of the Edinburgh Handedness Inventory: A one-factor congeneric measurement model using confirmatory factor analysis. Laterality, 9(4), 411-419. doi:10.1080/13576500342000248
Dragovic, M., \& Hammond, G. (2005). Handedness in schizophrenia: A quantitative review of evidence. Acta Psychiatrica Scandinavica, 111, 410-419. doi:10.1111/ j.1600-0447.2005.00519.x

Dragovic, M., Milenkovic, S., \& Hammond, G. (2008). The distribution of hand preference is discrete: A taxometric examination. British Journal of Psychology, 99, 445-459. doi:10.1348/000712608X304450

Edlin, J. M., Leppanen, M. L., Fain, R. J., \& Hackländer, R. P. (2015). On the use (and misuse?) of the Edinburgh Handedness Inventory. Brain and Cognition, 94, 44-51. doi:10.1016/j.bandc.2015.01.003

Fagard, J., \& Dahmen, R. (2010). Cultural influences on the development of lateral preferences: A comparison between French and Tunisian children. Laterality, 9(1), 67-78. doi:10.1080/13576500342000167

Faul, F., Erdfelder, E., Lang, A. G., \& Buchner, A. (2007a). G*Power 3: A flexible statistical power analysis program for the social, behavioral, and biomedical sciences. Behavior Research Methods, 39, 175-191. doi:10.3758/bf03193146

Faul, F., Erdfelder, E., Lang, A. G., \& Buchner, A. (2007b). $G^{*}$ Power 3 (Version 3.1.9.2 for Windows 10) [Computer Program]. Düsseldorf, Germany: Heinrich-HeineUniversität Düsseldorf. Retrieved from http://www. gpower.hhu.de/en.html.

Fazio, R., Coenen, C., \& Denney, R. L. (2012). The original instructions for the Edinburgh handedness inventory are misunderstood by a majority of participants. Laterality, 17(1), 70-77. doi:10.1080/1357650X.2010.532801

Fazio, R. L., \& Cantor, J. M. (2015). Factor structure of the Edinburgh handedness inventory versus the Fazio laterality inventory in a population with established atypical handedness. Applied Neuropsychology: Adult, 9095(April), 1-5. doi:10.1080/23279095.2014.940043

Fazio, R. L., Lykins, A. D., \& Cantor, J. M. (2014). Elevated rates of atypical handedness in paedophilia: Theory and implications. Laterality, 19(6), 690-704. doi:10.1080/ 1357650X.2014.898648

Fornell, C., \& Larcker, D. (1981). Structural equation models with unobservable variables and measurement error. Journal of Marketing Research, 18, 39-50.

Ghirlanda, S., \& Vallortigara, G. (2004). The evolution of brain lateralization: A game-theoretical analysis of population structure. Proceedings. Biological Sciences/The Royal Society, 271(1541), 853-857. doi:10.1098/rspb. 2003.2669

Goez, H., \& Zelnik, N. (2007). Handedness in patients with developmental coordination disorder. Journal of Child Neurology, 23(2), 151-154. doi:10.1177/0883073807307978

Greenwood, J. G., Greenwood, J. J., McCullagh, J. F., Beggs, J., \& Murphy, C. A. (2006). A survey of sidedness in Northern Irish school children: The interaction of sex, age and task. Laterality, 12(1), 1-18. doi:10.1080/13576500600886630

Hardie, S. M., \& Wright, L. (2014). Differences between leftand right-handers in approach/avoidance motivation: Influence of consistency of handedness measures. Frontiers in Psychology, 5, 134. doi:10.3389/fpsyg.2014.00134

Hill, M. M., \& Hill, A. (2000). Investigação por questionário [Research by questionnaire] (1st ed.). Lisboa, Portugal: Edições Sílabo.

Hu, L., \& Bentler, P. M. (1999). Cutoff criteria for fit indexes in covariance structure analysis: Conventional criteria 
versus new alternatives. Structural Equation Modeling, 6(1), 1-55. doi:10.1080/10705519909540118

Instituto Nacional de Estatística. (2014). Estatísticas Demográficas 2011 [Demographic Statistics 2011]. Lisboa, Portugal: INE.

Jöreskog, K. G. (1971a). Simultaneous factor analysis in several populations. Psychometrika, 36, 409-426. doi:10.1007/BF02291366

Jöreskog, K. G. (1971b). Statistical analysis of sets of congeneric test. Psychometrika, 36, 109-133. doi:10.1007/ BF02291393

Jöreskog, K. G. (1994). On the estimation of polychoric correlations and their asymptotic covariance matrix. Psychometrika, 59, 381-389. doi:10.1007/BF02296131

Kim, H. Y. (2013). Statistical notes for clinical researchers: Assessing normal distribution. Restorative Dentistry \& Endodontics, 38(1), 52-54. doi:10.5395/rde.2013.38.1.52

Lai, A., Serra, M., Petretto, D. R., Masala, C., \& Preti, A. (2014). Patterns of hand preference in Italian adolescent high-school students. Laterality, 19(6), 718-744. doi:10.1080/1357650X.2014.911747

Lange, N., DuBray, M. B., Lee, J. E., Froimowitz, M. P., Froehlich, A., Adluru, N., ... Lainhart, J. E. (2010). A typical diffusion tensor hemispheric asymmetry in autism. Autism Research, 3(6), 350-358. doi:10.1002/aur.162

Leask, D. S. J., \& Beaton, A. A. (2007). Handedness in Great Britain. Laterality, 12(6), 559-572. doi:10.1080/ 13576500701541936

950 Marôco, J. (2011). Análise Estatística com o SPSS statistics [Statistical analysis with SPSS statistics] (5th ed.). Pêro Pinheiro, Portugal: ReportNumber.

Martin, M., Papadatou-Pastou, M., Jones, G. V., \& Munafò, M. R. (2010). Sex and location as determinants of handedness: Reply to Vuoksimaa and Kaprio. Psychological Bulletin, 136, 348-350.

McFarland, K., \& Anderson, J. L. (1980). Factor stability of the Edinburgh Handedness Inventory as a function of testretest performance, age and sex. British Journal of Psychology, 71(1), 135-142. doi:10.1111/j.2044-8295.1980. tb02739.x

McManus, I. C., Moore, J., Freegard, M., \& Rawles, R. (2010). Science in the making: Right hand, left hand. III: Estimating historical rates of left-handedness. Laterality, 15, 186-208. doi:10.1080/13576500802565313

Medland, E. S., Perelle, I., Monte De, V., \& Ehrman, L. (2004). Effects of culture, sex, and age on the distribution of handedness: An evaluation of the sensitivity of three measures of handedness. Laterality, 9(3), 287-297.

970 Medland, S. E., Duffy, D. L., Wright, M. J., Geffen, G. M., Hay, D. A., Levy, F., ... Hewitt, A. W. (2009). Genetic influences on handedness: data from 25,732 Australian and Dutch twin families. Neuropsychologia, 47(2), 330-337. doi:10.1016/j.neuropsychologia.2008.09.005

975 Merni, F., Di Michele, R., \& Soffritti, G. (2014). Assessment of handedness using latent class factor analysis. Laterality, 19(4), 405-423. doi:10.1080/1357650X.2013. 845572

Messinger, H. B., \& Messinger, M. I. (1995). Factoring handedness data: I. Item analysis. Cortex, 31(4), 757-765. doi:10.1016/S0010-9452(13)80026-3

Milenkovic, S., \& Dragovic, M. (2013). Modification of the Edinburgh Handedness Inventory: A replication study.
Laterality, $\quad$ 18(3), $\quad 340-348 . \quad$ doi:10.1080/1357650X. 2012.683196

Millsap, R. E. (2012). Statistical approaches to measurement invariance. New York, NY: Routledge.

Oldfield, R. C. (1971). The assessment and analysis of handedness: The Edinburgh Inventory. Neuropsychologia, 9(1), 97-113. doi:10.1016/0028-3932(71)90067-4

Pallant, J. (2011). SPSS survival manual: A step by step guide to data analysis using SPSS for Windows (4th ed.). Crows Nest NSW, Australia: Allen \& Unwin.

Papadatou-Pastou, M., Martin, M., Munafò, M. R., \& Jones, G. V. (2008). Sex differences in left-handedness: A metaanalysis of 144 studies. Psychological Bulletin, 134(5), 677-699. doi:10.1037/a0012814

Payne, M. A. (1987). Impact of cultural pressures on selfreports of actual and approved hand use. Neuropsychologia, 25(1 PART 2), 247-258. doi:10.1016/0028-3932(87)90135-7

Perelle, I. B., \& Ehrman, L. (1994). An international study of human handedness: The data. Behavior Genetics, 24(3), 217-227. doi:10.1007/BF01067189

Pestana, M. H., \& Gageiro, J. N. (2008). Análise de dados para ciências sociais; A complementaridade do SPSS [Data analysis for the social sciences; Complementarity SPSS] (5th ed.). Lisboa, Portugal: Edições Sílabo.

Pogetti, L. S., De Souza, R. M., Tudella, E., \& Teixeira, L. A. (2013). Visibilidade dos braços afeta a preferência manual em bebés [Visibility of arms affect the hand preference in infants]. Motriz. Revista de Educação Física, 19(1), 160-170. doi:10.1590/S1980-65742013000100016

PORDATA. (2015). População residente com 15 e mais anos segundo os Censos: total e por nivel de escolaridade completo mais elevado [Resident population aged 15 and over, according to the Census: total and by highest level of educational qualifications obtained]. Retrieved from http://www. pordata.pt/Municipios/População+residente+com $+15+\mathrm{e}+$ mais+anos+segundo+os+Censos+total + e + por $+n \%$ C $3 \%$ ADvel+de+escolaridade+completo+mais+elevado-69-257.

Preti, A., Sisti, D., Rocchi, M. B., Busca, M., Vellante, M., Camboni, M. V., ... Masala, C. (2011). Male-female differences in left-handedness in Sardinia, Italy. Laterality, 16(6), 737-752. doi:10.1080/1357650X.2010.515991

Razali, N. M., \& Wah, Y. B. (2011). Power comparisons of Shapiro-Wilk, Kolmogorov-Smirnov, Lilliefors and Anderson-Darling tests. Journal of Statistical Modeling and Analytics, 2, 21-33.

Richardson, J. T. E. (1978). A factor analysis of selfreported handedness. Neuropsychologia, 16(6), 747-748. doi:10.1016/0028-3932(78)90010-6

Salmaso, D., \& Longoni, A. M. (1985). Problems in the assessment of hand preference. Cortex, 21(4), 533-549. doi:10.1016/S0010-9452(58)80003-9

Satz, P., Nelson, L., \& Green, M. (1989). Ambiguoushandedness: Incidence in a non-clinical sample. Neuropsychologia, 27(10), 1309-1310.

Shaw, J., Claridge, G., \& Clark, K. (2001). Schizotypy and the shift from dextrality: A study of handedness in a large non-clinical sample. Schizophrenia Research, 50(3), 181-189. doi:10.1016/S0920-9964(00)00167-5

Soper, H. V., \& Satz, P. (1984). Pathological left-handedness and ambiguous handedness: A new explanatory model. Neuropsychologia, 22(4), 511-515. doi:10.1016/0028-3932 (84)90046-0 
Souza, R. M., \& Teixeira, L. A. (2011). Sobre a relação entre filogenia e ontogenia no desenvolvimento da lateralidade na infância [On the relationship between phylogeny and ontogeny in the development of laterality in childhood]. Psicologia: Reflexão e Crítica, 24(1), 62-70. doi:10.1590/ S0102-79722011000100008

Tsuang, H. C., Chen, W. J., \& Kuo, S. Y. (2013). The crosscultural nature of the relationship between schizotypy and mixed-handedness. Laterality, 18(4), 476-490. doi:10.1080/1357650X.2012.720985

Valentini, F., \& Damásio, B. F. (2016). Variância média extraída e confiabilidade composta: Indicadores de precisão [Extracted and composite reliability: Reliability coefficients]. Psicologia: Teoria e Pesquisa, 32(2), e322225. doi:10.1590/0102-3772e322225

van Strien, J. W. (2002). The Dutch Handedness Questionnaire. Retrieved from http://hdl.handle.net/1765/956

Veale, J. F. (2014). Edinburgh Handedness Inventory - short form: A revised version based on confirmatory factor analysis. Laterality, 19(2), 164-77. doi:10.1080/1357650X.2013.783045 Viggiano, M. P., Borelli, P., Vannucci, M., \& Rocchetti, G. (2001). Hand preference in Italian students. Laterality, 6(3), 283-286. doi:10.1080/713754412

Williams, S. M. (1986). Factor analysis of the Edinburgh Handedness Inventory. Cortex, 22(2), 325-326. doi:10.1016/S0010-9452(86)80058-2 